\title{
Pricing formula for power options with jump-diffusion
}

\begin{abstract}
Payoff of a power option is typified by its underlying share price raised to a constant power. Also known as leveraged option, a minor change in its underlying may lead to a significant change in its price. In this study, we derive pricing formula for power options using the martingale approach when the underlying asset follows a jump-diffusion process.
\end{abstract}

Keyword: Power option; Leveraged option; Black-Scholes; Geometric Brownian motion; Jump-diffusion 